\title{
Quality of complementary and alternative medicine information for type 2 diabetes: a cross-sectional survey and quality assessment of websites
}

\author{
Jeremy Y. $\mathrm{Ng}^{*}$ (D), Manav Nayeni(D) and Kevin Gilotra
}

\begin{abstract}
Background: The global prevalence of diabetes mellitus is projected to reach approximately 700 million by the year 2045, with roughly 90-95\% of all diabetes cases being type 2 in nature. Patients with type 2 diabetes mellitus (T2DM) frequently seek information about complementary and alternative medicine (CAM) online. This study assessed the quality of publicly accessible websites providing consumer health information at the intersection of T2DM and CAM.

Methods: An online search engine (Google) was searched to identify pertinent websites containing information specific to CAM for T2DM patients, and the relevant websites were then screened with an eligibility criteria. Consumer health information found on eligible websites were then assessed for quality using the DISCERN instrument, a 16-item standardized scoring system.

Results: Across the 480 webpages identified, 94 unique webpages remained following deduplication, and 37 eligible webpages belonged to and were collapsed into 30 unique websites that were each assessed using the DISCERN instrument. The mean overall quality score (question 16) across all 30 assessed websites was 3.55 (SD = 0.86), and the mean summed DISCERN score was 52.40 ( $S D=12.11$ ). Eighty percent of websites presented a wide range of CAM treatment options with the associated benefits/risks of each treatment, but in $56.7 \%$ of the websites, the sources used to collect information were unreliable.

Conclusion: This study identified, assessed, and presents findings on the quality of online CAM information for T2DM. Although there were several high scoring websites, there was variability across most of the individual DISCERN items in the assessed websites. This study highlights the importance of awareness among healthcare providers regarding the reliability of online information about CAM treatment and management options for T2DM. Healthcare providers should be aware of patients' information seeking behaviour, guide them in navigating through the content they encounter online, and provide them with resources containing trustworthy and reliable information.
\end{abstract}

Keywords: Complementary and alternative medicine, Consumer health information, Diabetes, DISCERN, Information assessment, Quality of information, Type 2 diabetes mellitus

\footnotetext{
*Correspondence: ngjy2@mcmaster.ca

Department of Health Research Methods, Evidence, and Impact, Faculty of Health Sciences, Michael G. DeGroote Centre for Learning and Discovery, McMaster University, Room 2112, 1280 Main Street West, Hamilton, Ontario L8S 4K1, Canada
}

C C The Author(s). 2021 Open Access This article is licensed under a Creative Commons Attribution 4.0 International License, which permits use, sharing, adaptation, distribution and reproduction in any medium or format, as long as you give appropriate credit to the original author(s) and the source, provide a link to the Creative Commons licence, and indicate if changes were made. The images or other third party material in this article are included in the article's Creative Commons licence, unless indicated otherwise in a credit line to the material. If material is not included in the article's Creative Commons licence and your intended use is not permitted by statutory regulation or exceeds the permitted use, you will need to obtain permission directly from the copyright holder. To view a copy of this licence, visit http://creativecommons.org/licenses/by/4.0/ The Creative Commons Public Domain Dedication waiver (http://creativecommons.org/publicdomain/zero/1.0/) applies to the data made available in this article, unless otherwise stated in a credit line to the data. 


\section{Background}

According to the International Diabetes Federation, the global prevalence of diabetes mellitus was approximately 439 million in 2019 [1]. Type 2 diabetes mellitus (T2DM) roughly accounts for $90-95 \%$ of all cases [2]. The global prevalence of diabetes mellitus is projected to reach approximately 700 million by the year 2045 [1]. T2DM is a metabolic disorder characterized by insulin resistance, which refers to a diminished response to the hormone insulin in the body [3]. Insulin plays a crucial role in glucose homeostasis by reducing blood glucose levels [4]. Patients with T2DM typically display elevated blood glucose levels [5]. Treatment for T2DM typically includes lifestyle changes as well as the use of oral hypoglycaemics to promote glucose uptake $[5,6]$. The chronic nature of T2DM can have adverse physiological effects on individuals over time including retinopathy, neuropathy and diabetic ulcers [3].

Patients diagnosed with chronic conditions, including T2DM, often seek complementary or alternative medicine (CAM) to manage their health [7]. As an example, it has been found that over $79 \%$ of Canadians had used CAM at some point in their life [8]. CAM refers to practices and therapies that are not extensively taught in Western medical schools nor typically utilized in conventional medicine; specifically, "complementary medicine" includes non-conventional practices used in conjunction with conventional care, while "alternative medicine" refers to nonconventional practices used in replacement of conventional ones [9-11]. Some examples of frequently used CAM therapies for T2DM include acupuncture, mindbody interventions, and dietary supplements [12, 13]. According to a 2018 nationwide survey within the United States, 57\% of patients with T2DM have used CAM [13]. Oftentimes, patients consult the internet as an avenue for identifying potential CAM treatment options for their health conditions [14]. The internet is the primary source of CAM information for many patients, making it important to determine the quality of the information accessible to the general public [14]. To our knowledge, no prior research has identified nor assessed the quality of consumer health information presented online for CAM-specific treatment and/or management options for T2DM, thus this is the purpose of this study.

\section{Methods}

\section{Search strategy and screening}

A search strategy was developed to yield websites; Google was the sole websites searched, as it is the most commonly used search engine globally [15]. The search terms we selected were designed to reflect a "typical" patient's information-seeking behaviour regarding CAM for T2DM. Four search terms were searched as follows: "alternative medicine for diabetes", "complementary and alternative medicine for diabetes", "complementary medicine for diabetes" and "integrative medicine for diabetes". Searches were conducted using the Google Chrome browser in incognito mode, to ensure that searches were not affected by previous browser search histories. Through the search settings, the Google location was changed to include results from Australia (Google.com.au), Canada (Google.ca), the United Kingdom (Google.co.uk) and the United States (Google.com), to reflect a more internationally representative sample of websites.

\section{Eligibility criteria}

Websites were screened for eligibility and were included if they contained CAM consumer health information pertaining to the treatment and/or management of T2DM. KG and another research assistant screened all results from the first two Google pages for each search term, and duplicate websites were removed. The websites also had to be freely available to the general public and written in the English language. Websites were excluded if they were peer-reviewed journals or articles, websites of major consumer retailers (eBooks, Google Books, etc.) as these are pay-to-view, were broken URLs, were from online newspapers that did not provide consumer health information or were exclusively video sites (i.e., YouTube). Different webpages captured from the same website were collapsed into a single item, as we applied the DISCERN instrument to information found across websites, and not individual webpages [16].

\section{Data extraction and website quality assessment}

KG and the other research assistant extracted the following background information from eligible websites: the website name and URL, types of CAM and non-CAM therapies mentioned, whether the website appeared in more than one search and whether the website was certified by Health on the Net Foundation Code of Conduct (HONcode). We also classified each eligible website into one of five categories as follows: health portal, professional, news, government or other. The professional category encompassed websites developed by healthcare professionals/experts or health organizations. The health portal category included websites that presented information about a range of health topics. The government category included websites belonging to official government organizations and bodies. The news category consisted of websites from media sources such as newspapers, magazines or television stations and that were created for the distribution of current events. Lastly, the other category included any other websites that did not fall into any of the previously listed categories. We then assessed the quality of consumer health information available on each eligible website using the 
DISCERN instrument. The DISCERN instrument is comprised of 16 items divided into three sections, each of which aims to assess the quality of written consumer health information [16]. The first section, comprised of questions $1-8$, assesses the reliability of the information presented. The second section, comprised of questions 9-15, assesses the quality of the information presented as treatment choices. Lastly, the third section is comprised of a singular question, question 16, and asks the user to provide an overall rating for the resource in its entirety. Each individual question was scored on a Likert scale from one to five, whereby one represented the lowest quality and five the highest quality [16].

To standardize the scoring process using the DISCERN instrument, JYN, KG and the other research assistant conducted a pilot test on three separate websites and used the DISCERN instrument to score the websites. Following the pilot test, all three assessors met to compare their scores and resolve any discrepancies to ensure the standardization of scores and that the DISCERN instrument was applied accurately according to the manual's instructions. Following the standardization, KG and the other research assistant scored each eligible website using the instrument. Following scoring, JYN met with the two assessors and all discrepancies between the scores of the two assessors were resolved, without unduly modifying scores. MN averaged the two assessors' scores for each question across all eligible websites, and a summed DISCERN score between values 15-75 was tabulated from questions 1-15. MN then calculated the average and standard deviation for each score, alongside an average score for all 16 items, and JYN reviewed all calculations. It is important to note that while the DISCERN instrument can be used to judge the reliability of a website as a source of information about treatment choices, it cannot be used to assess the scientific quality or accuracy of the evidence on which a publication is based, as this would require checking the information presented on each website against other sources [16].

\section{Results}

\section{Search results}

Of the 480 webpages returned from the Google searches, 94 unique webpages remained following deduplication. Of these, 64 webpages were excluded for the following reasons: were peer-reviewed articles $(n=40)$, did not discuss any CAM therapies and/or recommendations $(n=$ $11)$, were webpages from the same website $(n=7)$, were video websites $(n=3)$, did not discuss diabetes $(n=2)$, or was a news website that did not provide consumer health information $(n=1)$. The remaining 30 websites were then assessed using the DISCERN instrument. A flowchart of the search and screening process illustrated in Fig. 1.

\section{General characteristics of eligible websites}

We categorized the 30 eligible websites as follows: health portals $(n=13)$, professional $(n=12)$, news (either online magazines/TV channels) $(n=3)$, government $(n=1)$, and other $(n=1)$. Commonly mentioned CAM therapies across websites included the following: acupuncture $(n=$ $12)$, chromium $(n=11)$, yoga $(n=9)$, magnesium $(n=6)$ and aloe vera $(n=5)$. The most common non-CAM therapies mentioned across the websites included selective sodium-glucose transporter- 2 inhibitors $(n=3)$, thiazolidinediones $(n=2)$, dipeptidyl peptidase-4 inhibitors $(n=2)$ and dietary monitoring $(n=2)$. A detailed account of the general characteristics of the eligible websites are shown in Table 1.

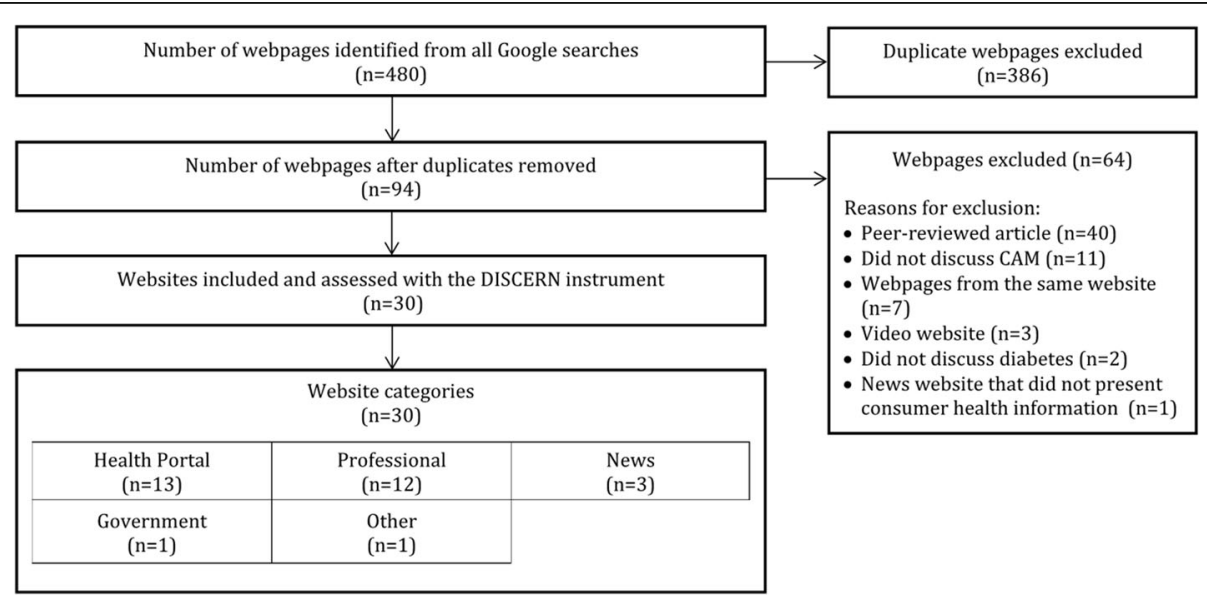

Fig. 1 Web Information Search Strategy and Assessment Flowchart 
Table 1 General characteristics of eligible websites

\begin{tabular}{|c|c|c|c|c|c|}
\hline $\begin{array}{l}\text { Website } \\
\text { Name }\end{array}$ & URL & $\begin{array}{l}\text { Website } \\
\text { Category }\end{array}$ & Types of CAM Discussed & $\begin{array}{l}\text { Types of Non-CAM Therapies } \\
\text { Discussed }\end{array}$ & $\begin{array}{l}\text { Appeared } \\
\text { in More } \\
\text { than One } \\
\text { Search? } \\
\end{array}$ \\
\hline Alt-Med & http://www.alt-med.org & Professional & $\begin{array}{l}\text { Select supplement and herbs, stress } \\
\text { reduction therapies, reasonable } \\
\text { lifestyle changes }\end{array}$ & None & No \\
\hline $\begin{array}{l}\text { Association of } \\
\text { Traditional } \\
\text { Medicine and } \\
\text { Acupuncture } \\
\text { UK }\end{array}$ & https://www.atcm.co.uk & Professional & $\begin{array}{l}\text { Acupuncture, huangqi, lugen, } \\
\text { tianhaufen, huangbai, zexie, } \\
\text { danzhuyet }\end{array}$ & None & No \\
\hline $\begin{array}{l}\text { Canadian } \\
\text { College of } \\
\text { Naturopathic } \\
\text { Medicine }\end{array}$ & https://rsnc.ca & Professional & $\begin{array}{l}\text { Chromium, homeopathy, traditional } \\
\text { Chinese medicines, botanical } \\
\text { medicine }\end{array}$ & None & No \\
\hline Chelation & https://chelation.me & Other & $\begin{array}{l}\text { Gluten free diet, coffee, oolong tea, } \\
\text { yoga, tai chi, meditation, vitamin C, } \\
\text { magnesium, chromium, biotin, } \\
\text { vitamin D, alpha lipoic acid, bitter } \\
\text { melon, fenugreek, G. sylvestre, } \\
\text { cinnamon, Gui zhi, ginseng, berberine, } \\
\text { Gaertn sulyonarin, Shenyan kangfu }\end{array}$ & $\begin{array}{l}\text { Dietary monitoring, } \\
\text { thiazolidinediones, dipeptidyl } \\
\text { peptidase-4 inhibitors, selective } \\
\text { sodium-glucose transporter inhibitors, } \\
\text { insulin, chelation therapy }\end{array}$ & No \\
\hline $\begin{array}{l}\text { Conscious } \\
\text { Lifestyle } \\
\text { Magazine }\end{array}$ & $\begin{array}{l}\text { https://www. } \\
\text { consciouslifestylemag. } \\
\text { com }\end{array}$ & $\begin{array}{l}\text { Online } \\
\text { magazine }\end{array}$ & $\begin{array}{l}\text { Essential dietary oils, polyphenols, } \\
\text { chromium, magnesium, R- alpha } \\
\text { lipoic acid, botanical medicines, G. } \\
\text { sylvestre, cinnamon, green tea leaf } \\
\text { extract, curcumin extract }\end{array}$ & None & No \\
\hline $\begin{array}{l}\text { Diabetes } \\
\text { Australia }\end{array}$ & $\begin{array}{l}\text { https://www. } \\
\text { diabetesaustralia.com.au }\end{array}$ & Government & Homeopathy, guanmu tong & None & No \\
\hline $\begin{array}{l}\text { Diabetes in } \\
\text { Control }\end{array}$ & $\begin{array}{l}\text { http://www. } \\
\text { diabetesincontrol.com }\end{array}$ & Professional & $\begin{array}{l}\text { Herbal therapies, chiropractic therapy, } \\
\text { massage therapy, acupuncture, yoga, } \\
\text { meditation }\end{array}$ & None & No \\
\hline $\begin{array}{l}\text { Diabetes Self } \\
\text { Management }\end{array}$ & $\begin{array}{l}\text { https://www. } \\
\text { diabetesselfmanagement. } \\
\text { com }\end{array}$ & $\begin{array}{l}\text { Health } \\
\text { Portal }\end{array}$ & $\begin{array}{l}\text { Acupuncture, hypnosis, acupressure, } \\
\text { qigong, mindfulness, ayurveda }\end{array}$ & None & No \\
\hline Diabetes.co.uk & $\begin{array}{l}\text { https://www.diabetes.co. } \\
\text { uk }\end{array}$ & $\begin{array}{l}\text { Health } \\
\text { Portal }\end{array}$ & $\begin{array}{l}\text { A. vera, bilberry extract, bitter melon, } \\
\text { cinnamon, fenugreek, Ginger, okra } \\
\text { allium, B. forficata, Myrcia uniflora, C. } \\
\text { indica, F. carica, ginseng, G. sylvestre, } \\
\text { M. charantia, O. sanctum, O. } \\
\text { streptacantha, Trigonella foeunum } \\
\text { graecum, berberine cinnamomum, } \\
\text { tamala curry, E. jambolana, gingko, P. } \\
\text { amarus, P. marsupium, S. torvum, V. } \\
\text { rosea }\end{array}$ & None & Yes \\
\hline $\begin{array}{l}\text { Diabetic } \\
\text { Nation }\end{array}$ & $\begin{array}{l}\text { https://diabeticnation. } \\
\text { com }\end{array}$ & $\begin{array}{l}\text { Health } \\
\text { Portal }\end{array}$ & $\begin{array}{l}\text { Herbal therapy, aromatherapy, yoga, } \\
\text { meditation, acupuncture, acupressure, } \\
\text { massage therapy }\end{array}$ & None & No \\
\hline $\begin{array}{l}\text { Dr. Kirsten } \\
\text { Smith, ND }\end{array}$ & $\begin{array}{l}\text { https://www.drsmithnd. } \\
\text { com }\end{array}$ & Professional & $\begin{array}{l}\text { Botanical medicine, traditional } \\
\text { Chinese medicines }\end{array}$ & None & No \\
\hline Dr. Axe & https://draxe.com & Professional & $\begin{array}{l}\text { Chromium picolinate, magnesium, } \\
\text { healthy fats, low glycemic diet, } \\
\text { cinnamon, fish oil, alpha lipoic acid, } \\
\text { bitter melon, exercise, yoga }\end{array}$ & None & No \\
\hline EndocrineWeb & $\begin{array}{l}\text { https://www. } \\
\text { endocrineweb.com }\end{array}$ & Professional & $\begin{array}{l}\text { Low glycaemic diet, chromium, stress } \\
\text { reduction, acupuncture, alpha lipoic } \\
\text { acid, omega-3 fatty acids, polyphenols }\end{array}$ & None & No \\
\hline $\begin{array}{l}\text { Endocrinology } \\
\text { Network }\end{array}$ & $\begin{array}{l}\text { https://www. } \\
\text { endocrinologynetwork. } \\
\text { com/view/alternative- } \\
\text { and-complementary- }\end{array}$ & Professional & $\begin{array}{l}\text { Chromium, G. sylvestre, bitter melon, } \\
\text { aloe vera, fenugreek, cinnamon, } \\
\text { vanadium, omega-3, vitamin D, acu- } \\
\text { puncture, tai chi, qigong, yoga, }\end{array}$ & None & Yes \\
\hline
\end{tabular}


Table 1 General characteristics of eligible websites (Continued)

\begin{tabular}{|c|c|c|c|c|c|}
\hline $\begin{array}{l}\text { Website } \\
\text { Name }\end{array}$ & URL & $\begin{array}{l}\text { Website } \\
\text { Category }\end{array}$ & Types of CAM Discussed & $\begin{array}{l}\text { Types of Non-CAM Therapies } \\
\text { Discussed }\end{array}$ & $\begin{array}{l}\text { Appeared } \\
\text { in More } \\
\text { than One } \\
\text { Search? }\end{array}$ \\
\hline & medicine-diabetes & & laughter therapy & & \\
\hline $\begin{array}{l}\text { Everyday } \\
\text { Health }\end{array}$ & $\begin{array}{l}\text { https://www. } \\
\text { everydayhealth.com }\end{array}$ & $\begin{array}{l}\text { Health } \\
\text { Portal }\end{array}$ & Exercise, yoga, stress management & Sleep apnea devices & Yes \\
\hline Healthline & $\begin{array}{l}\text { https://www.healthline. } \\
\text { com }\end{array}$ & $\begin{array}{l}\text { Health } \\
\text { Portal }\end{array}$ & $\begin{array}{l}\text { Exercise, low carb diet, stress } \\
\text { management, yoga, mindfulness } \\
\text { exercises, chromium, magnesium, } \\
\text { apple cider vinegar, cinnamon, } \\
\text { berberine, fenugreek }\end{array}$ & None & Yes \\
\hline $\begin{array}{l}\text { Institute for } \\
\text { Natural } \\
\text { Medicine }\end{array}$ & https://naturemed.org & $\begin{array}{l}\text { Health } \\
\text { Portal }\end{array}$ & Herbal substitutes & Insulin & No \\
\hline $\begin{array}{l}\text { Medical News } \\
\text { Today }\end{array}$ & $\begin{array}{l}\text { https://www. } \\
\text { medicalnewstoday.com }\end{array}$ & $\begin{array}{l}\text { Health } \\
\text { Portal }\end{array}$ & $\begin{array}{l}\text { A. vera, cinnamon, bitter melon, milk } \\
\text { thistle, fenugreek, gymnema, ginger }\end{array}$ & None & No \\
\hline NDTV Food & https://food.ndtv.com & $\begin{array}{l}\text { Online TV } \\
\text { station } \\
\text { website }\end{array}$ & $\begin{array}{l}\text { Fenugreek seeds, neem, turmeric } \\
\text { powder, bitter gourd, gurmar }\end{array}$ & None & Yes \\
\hline $\begin{array}{l}\text { On Track } \\
\text { Diabetes }\end{array}$ & $\begin{array}{l}\text { https://www. } \\
\text { ontrackdiabetes.com }\end{array}$ & $\begin{array}{l}\text { Health } \\
\text { Portal }\end{array}$ & $\begin{array}{l}\text { Homeopathy, acupuncture, } \\
\text { supplements, yoga, massage, organic } \\
\text { food diet }\end{array}$ & None & No \\
\hline $\begin{array}{l}\text { Onyx } \\
\text { Integrative } \\
\text { Medicine and } \\
\text { Aesthetics }\end{array}$ & $\begin{array}{l}\text { https://onyxintegrative. } \\
\text { com }\end{array}$ & Professional & $\begin{array}{l}\text { Acupuncture, mind-body medicine, } \\
\text { exercise, supplements }\end{array}$ & None & No \\
\hline Practo & https://www.practo.com & $\begin{array}{l}\text { Health } \\
\text { Portal }\end{array}$ & $\begin{array}{l}\text { Bitter gould, fenugreek, mango leaves, } \\
\text { Indian gooseberry, moringa leaves }\end{array}$ & None & No \\
\hline $\begin{array}{l}\text { Stamford } \\
\text { Health }\end{array}$ & $\begin{array}{l}\text { https://www. } \\
\text { stamfordhealth.org }\end{array}$ & Professional & $\begin{array}{l}\text { Apple cider vinegar, barley, } \\
\text { chromium, zinc, aloe vera, berberine, } \\
\text { cinnamon, fenugreek, gymnema, } \\
\text { nopal }\end{array}$ & None & No \\
\hline $\begin{array}{l}\text { The Health } \\
\text { Site }\end{array}$ & $\begin{array}{l}\text { https://www. } \\
\text { thehealthsite.com }\end{array}$ & $\begin{array}{l}\text { Health } \\
\text { Portal }\end{array}$ & $\begin{array}{l}\text { Tulsi leaves, flax seeds, bilberry leaves, } \\
\text { cinnamon, green tea, drumstick } \\
\text { leaves, psyllium husk, bitter gourd, } \\
\text { neem, Indian blackberry }\end{array}$ & None & No \\
\hline $\begin{array}{l}\text { Today's } \\
\text { Dietitian }\end{array}$ & $\begin{array}{l}\text { https://www. } \\
\text { todaysdietitian.com }\end{array}$ & $\begin{array}{l}\text { Online } \\
\text { magazine }\end{array}$ & $\begin{array}{l}\text { Gluten free diet, fish oils, yoga, } \\
\text { qigong }\end{array}$ & None & No \\
\hline Totally Health & $\begin{array}{l}\text { https://www. } \\
\text { totallyhealth.com }\end{array}$ & $\begin{array}{l}\text { Health } \\
\text { Portal }\end{array}$ & $\begin{array}{l}\text { Acupuncture, Chinese herbal } \\
\text { medicines, Ayurvedic medicines, } \\
\text { vitamin D, aromatherapy, relaxation } \\
\text { therapy, guided imagery, massage } \\
\text { therapy, homeopathy, biofeedback }\end{array}$ & None & No \\
\hline $\begin{array}{l}\text { University of } \\
\text { Minnesota }\end{array}$ & $\begin{array}{l}\text { https://www. } \\
\text { takingcharge.csh.umn. } \\
\text { edu }\end{array}$ & Professional & $\begin{array}{l}\text { Acupuncture, moxibustion, Chinese } \\
\text { herbal medicines, diet therapy, } \\
\text { qigong, tai chi, tui na, P. ginseng, } \\
\text { Momodica charantia, Lageneria } \\
\text { siceraria, Psidium gnajava, alpha lipoic } \\
\text { acid, essential fatty acids, vitamin C, } \\
\text { ginkgo biloba, mind-body practices, } \\
\text { stress management }\end{array}$ & Insulin, exercise & No \\
\hline $\begin{array}{l}\text { University of } \\
\text { Rochester } \\
\text { Medical Centre }\end{array}$ & $\begin{array}{l}\text { https://www.urmc. } \\
\text { rochester.edu }\end{array}$ & Professional & $\begin{array}{l}\text { Alpha lipoic acid, chromium, } \\
\text { polyphenols, ginseng, acupuncture, } \\
\text { exercise }\end{array}$ & $\begin{array}{l}\text { Selective sodium-glucose transporter } \\
2 \text { inhibitors }\end{array}$ & No \\
\hline $\begin{array}{l}\text { Very Well } \\
\text { Health }\end{array}$ & $\begin{array}{l}\text { https://www. } \\
\text { verywellhealth.com }\end{array}$ & $\begin{array}{l}\text { Health } \\
\text { Portal }\end{array}$ & $\begin{array}{l}\text { Ginseng, chromium, magnesium, } \\
\text { cinnamon, aloe vera, gymnema }\end{array}$ & $\begin{array}{l}\text { Exercise, portion control, insulin, } \\
\text { biguanides, thiazolidinediones, alpha- } \\
\text { glucosidase inhibitors. Meglitinides, } \\
\text { dipeptidyl peptidase-4 inhibitors, se- } \\
\text { lective sodium-glucose transporter in- } \\
\text { hibitors, incretin mimetics, amylin }\end{array}$ & No \\
\hline
\end{tabular}


Table 1 General characteristics of eligible websites (Continued)

\begin{tabular}{|c|c|c|c|c|c|}
\hline $\begin{array}{l}\text { Website } \\
\text { Name }\end{array}$ & URL & $\begin{array}{l}\text { Website } \\
\text { Category }\end{array}$ & Types of CAM Discussed & $\begin{array}{l}\text { Types of Non-CAM Therapies } \\
\text { Discussed }\end{array}$ & $\begin{array}{l}\text { Appeared } \\
\text { in More } \\
\text { than One } \\
\text { Search? }\end{array}$ \\
\hline & & & & analogue, bariatric surgery & \\
\hline WebMD & $\begin{array}{l}\text { https://www.webmd. } \\
\text { com }\end{array}$ & $\begin{array}{l}\text { Health } \\
\text { Portal }\end{array}$ & $\begin{array}{l}\text { Acupuncture, vanadium, magnesium, } \\
\text { ginseng, chromium, biofeedback, } \\
\text { guided imagery, chitosan, Garcinia } \\
\text { cambogia, pyruvate, germander, M. } \\
\text { charantia, S. androgynus, aristolochic } \\
\text { acid }\end{array}$ & Insulin & No \\
\hline
\end{tabular}

\section{DISCERN instrument ratings}

The average summed DISCERN score across the eligible 30 websites was $52.40(\mathrm{SD}=12.10)$. The average overall quality score for all the websites (question 16) was 3.55 $(\mathrm{SD}=0.86)$. The three websites with the highest DISCERN score were as follows: WebMD (69.00), Diabetes Self Management (67.50) and Diabetes.co.uk (66.50). These three websites scored consistent $4 \mathrm{~s}$ and $5 \mathrm{~s}$ across all 16 DISCERN items. The three poorest scoring websites were as follows: Onyx Integrative Medicine and Aesthetics (32.50), Canadian College of Naturopathic Medicine (32.00) and NDTV Food (31.00). These websites consistently scored $2 \mathrm{~s}$ and $3 \mathrm{~s}$ across most of the DISCERN items, performing particularly poorly in the first section of the DISCERN instrument, which focused on assessing the reliability of the information presented on the website. Across most of the websites, the majority performed better on the second section of the DISCERN instrument, which assesses the quality of the information presented on the website, compared to the first section. All DISCERN scores for each of the eligible websites can be found in Table 2 .

\section{Trends identified across resources assessed Reliability issues}

Question four of the DISCERN instrument assessed the reliability of the sources used to present information on the websites, particularly assessing whether the information provided by the website is supported by a source that readers can identify. Many eligible websites scored very poorly on this item. Of the 30 websites, 17 (56.7\%) of them scored a 3 or lower.

\section{Clear treatment options with benefits}

Questions nine and 10 of the DISCERN instrument assessed whether the websites thoroughly discussed how the individual treatments work, and if the benefits of each of the treatments are stated, respectively. The majority of the websites scored highly on these two questions, with 21 out of $30(70.0 \%)$ websites for question nine, and 23 out of $30(76.7 \%)$ websites for question 10 , scoring 4 or higher. Most of the websites presented many individual treatment options and listed the benefits of the treatments with explanations and evidence.

\section{Multiple treatment options presented}

Question 14 of the DISCERN instrument assessed whether the websites considered a variety of treatment options for T2DM. The majority of the websites scored highly on this question; out of the 30 websites, 24 (80\%) scored a 4 or higher for this item. Beyond presenting CAM options for the treatment and/or management of T2DM, the websites emphasized the importance of there being multiple treatment choices to manage T2DM symptoms and elaborated on different therapy options.

\section{Highly scoring websites for patients and consumers}

For the purpose of this study, we defined highly scoring websites are those with an overall DISCERN score of 60 or higher and that have a score of at least four out of the possible five for the mean of the first 15 questions of the instrument. Out of the 30 eligible websites, only 11 met this criteria. Nine of the 11 websites scored a 4.5 or higher for question 5, which focused on assessing whether the websites information was unbiased and balanced. Additionally, all 11 websites scored a 4.5 or higher on question 10 of the instrument, which focused on identifying whether the website presented all treatment options and described the benefits of each treatment. The highly scoring websites performed well on this question because they presented treatment options clearly, used subheadings for each treatment, and provided details under each subheading outlining the intended benefits of each treatment. Each highly scoring website also scored a 4.5 or higher on question 14, which assessed whether more than one treatment choice was presented. Detailed characteristics of these highly scoring websites are shown in Table 3.

\section{Discussion}

The purpose of this study was to identify and assess the quality of websites providing CAM consumer health information for the treatment and/or management of T2DM. Many patients consult online resources with the 


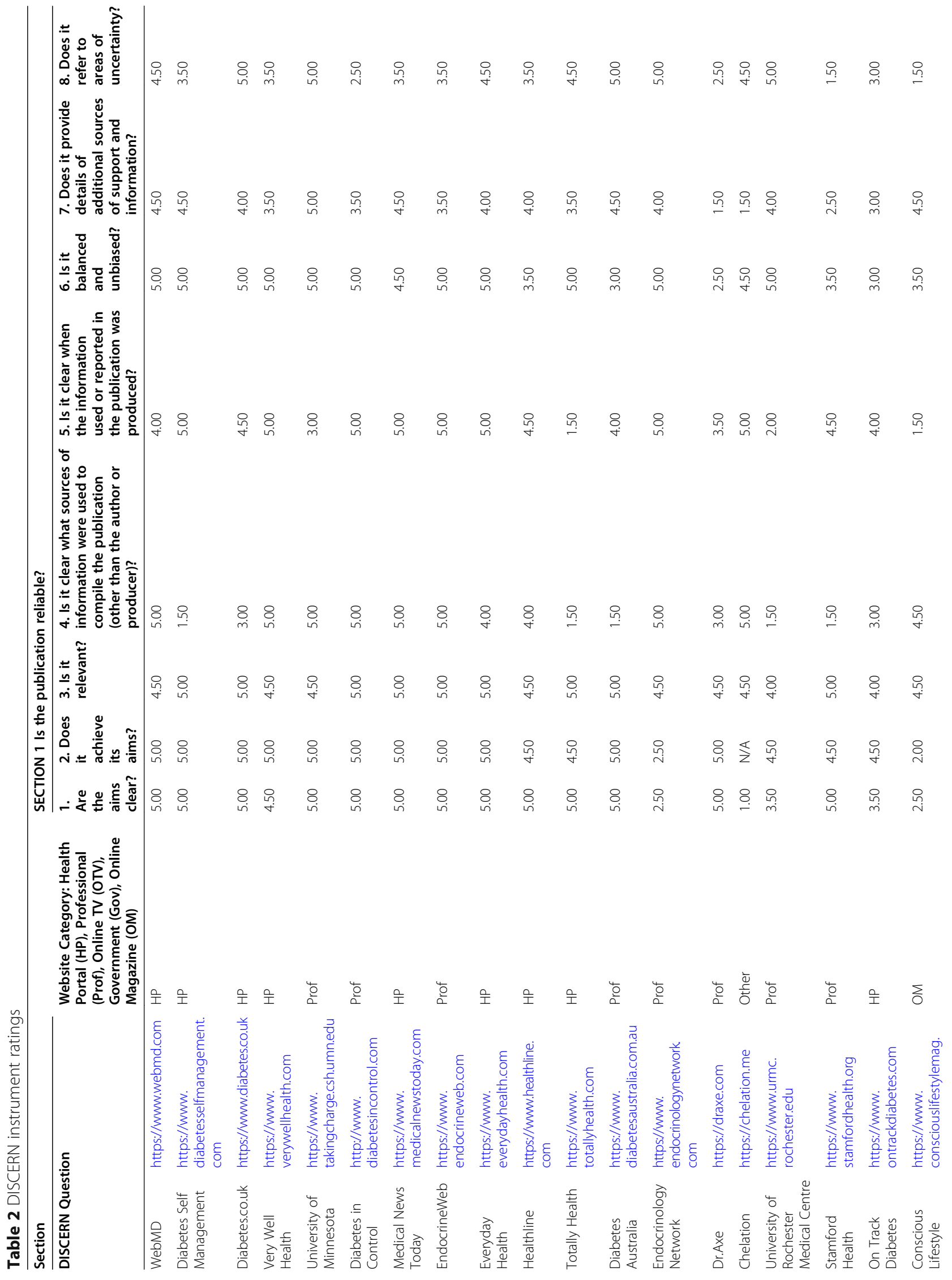




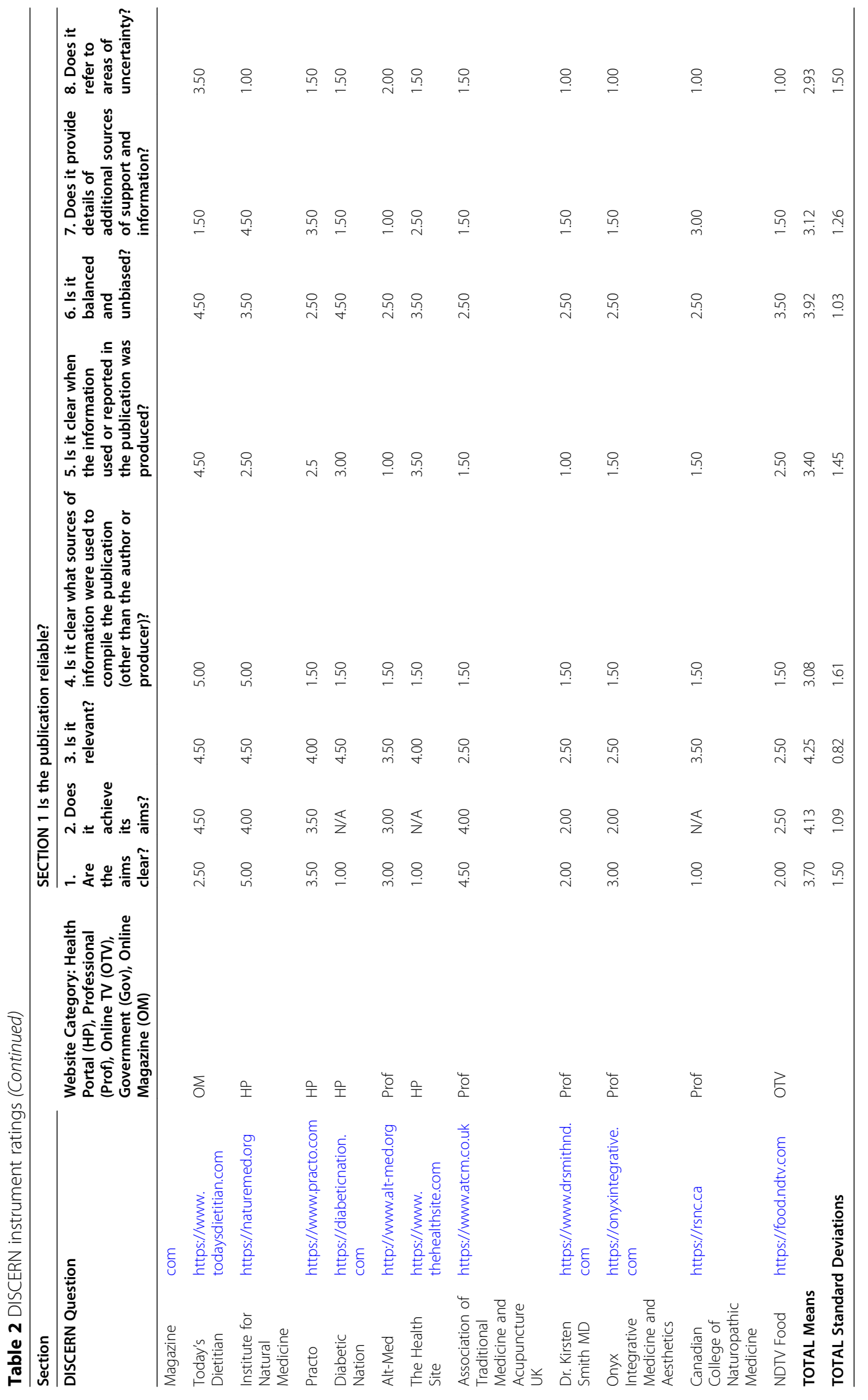




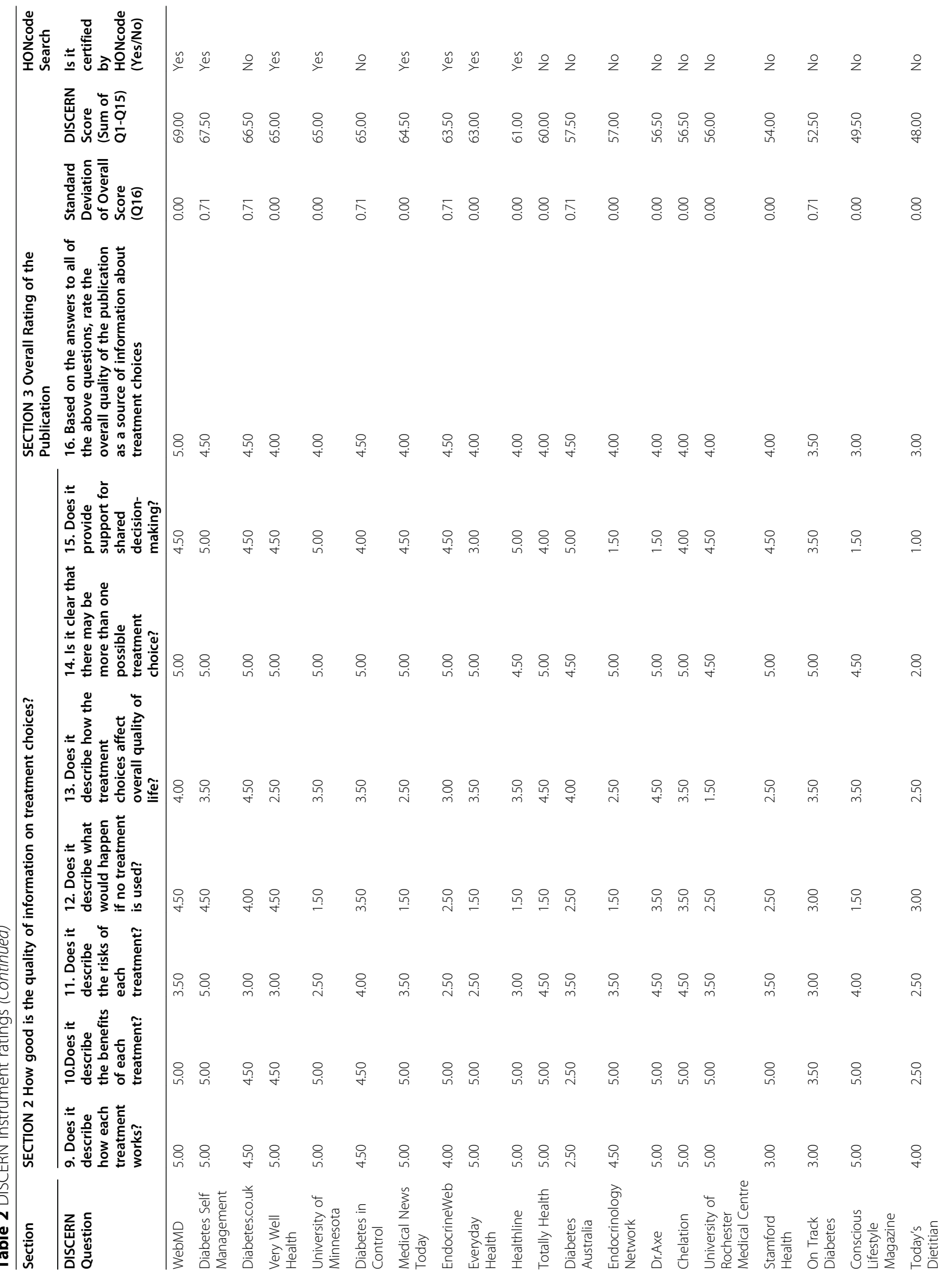



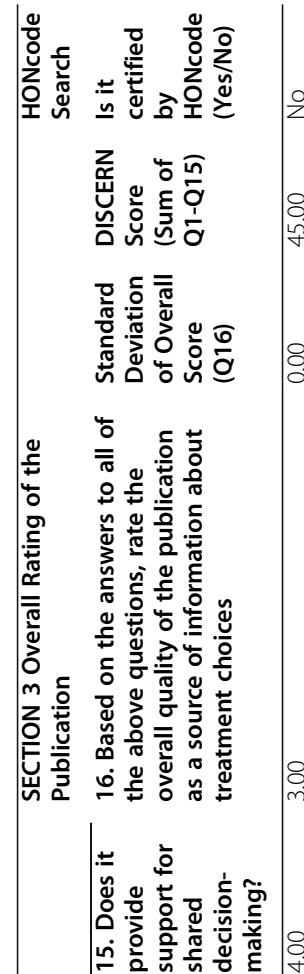

8.8

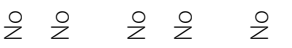

요 2

之

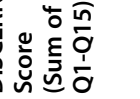

8.

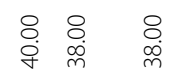

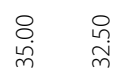

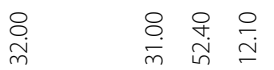

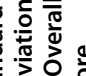

8

웅

ঃ.

8

웅 ㅎํㅇ

范

ไั่

نे

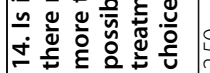

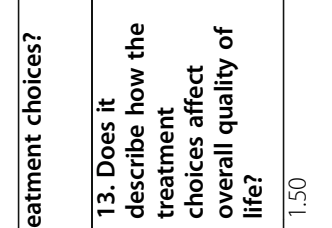

号号品号色

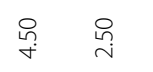

$\underset{+}{8}$

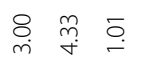

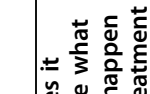

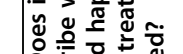

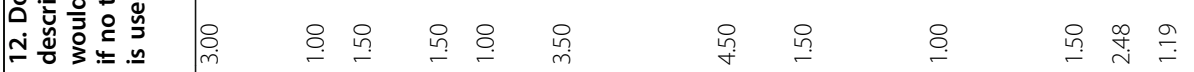

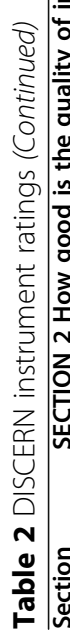

:

范

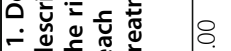

P.

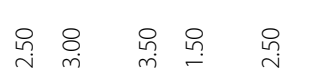

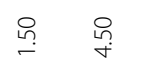

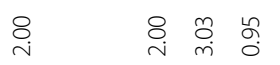

:

每

in

गु

$\underset{+}{8} \stackrel{\leftrightarrow}{\leftarrow}$

$\stackrel{\circ}{\text { ก }}$

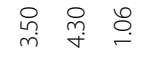

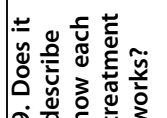

운

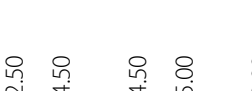

욜ํำ

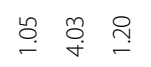

|

京 
Table 3 Highly scoring websites for patients and consumers

\begin{tabular}{|c|c|c|c|c|c|c|}
\hline $\begin{array}{l}\text { Website } \\
\text { Name }\end{array}$ & URL & $\begin{array}{l}\text { Summed DISCERN } \\
\text { Score (out of } 75 \text { total } \\
\text { points) }\end{array}$ & $\begin{array}{l}\text { Mean } \\
\text { DISCERN } \\
\text { Score for Q16 }\end{array}$ & $\begin{array}{l}\text { Website } \\
\text { Category }\end{array}$ & Target Audience & $\begin{array}{l}\text { Frequency of } \\
\text { Updates }\end{array}$ \\
\hline WebMD & $\begin{array}{l}\text { https://www.webmd.com/ } \\
\text { diabetes/natural-remedies- } \\
\text { type-2-diabetes }\end{array}$ & 69.00 & 5.00 & $\begin{array}{l}\text { Health } \\
\text { Portal }\end{array}$ & Patients/public & $\begin{array}{l}\text { Update } \\
\text { frequency not } \\
\text { available }\end{array}$ \\
\hline $\begin{array}{l}\text { Diabetes Self } \\
\text { Management }\end{array}$ & $\begin{array}{l}\text { https://www. } \\
\text { diabetesselfmanagement.com }\end{array}$ & 67.50 & 4.50 & $\begin{array}{l}\text { Health } \\
\text { Portal }\end{array}$ & $\begin{array}{l}\text { Healthcare providers, } \\
\text { patients/public }\end{array}$ & $\begin{array}{l}\text { Updated } \\
\text { occasionally }\end{array}$ \\
\hline Diabetes.co.uk & https://www.diabetes.co.uk & 66.50 & 4.50 & $\begin{array}{l}\text { Health } \\
\text { Portal }\end{array}$ & $\begin{array}{l}\text { Healthcare providers, } \\
\text { patients/public }\end{array}$ & $\begin{array}{l}\text { Updated } \\
\text { occasionally }\end{array}$ \\
\hline $\begin{array}{l}\text { Very Well } \\
\text { Health }\end{array}$ & https://www.verywellhealth.com & 65.00 & 4.00 & $\begin{array}{l}\text { Health } \\
\text { Portal }\end{array}$ & $\begin{array}{l}\text { Healthcare providers, } \\
\text { patients/public }\end{array}$ & $\begin{array}{l}\text { Website is } \\
\text { updated } \\
\text { monthly }\end{array}$ \\
\hline $\begin{array}{l}\text { University of } \\
\text { Minnesota }\end{array}$ & $\begin{array}{l}\text { https://www.takingcharge.csh. } \\
\text { umn.edu }\end{array}$ & 69.00 & 4.00 & Professional & $\begin{array}{l}\text { Healthcare providers, } \\
\text { researchers, patients/ } \\
\text { public }\end{array}$ & $\begin{array}{l}\text { Update } \\
\text { frequency not } \\
\text { available }\end{array}$ \\
\hline $\begin{array}{l}\text { Diabetes in } \\
\text { Control }\end{array}$ & $\begin{array}{l}\text { http://www.diabetesincontrol. } \\
\text { com }\end{array}$ & 67.50 & 4.50 & Professional & $\begin{array}{l}\text { Researchers, healthcare } \\
\text { professionals }\end{array}$ & $\begin{array}{l}\text { Website is } \\
\text { updated } \\
\text { occasionally }\end{array}$ \\
\hline $\begin{array}{l}\text { Medical News } \\
\text { Today }\end{array}$ & $\begin{array}{l}\text { https://www.medicalnewstoday. } \\
\text { com }\end{array}$ & 66.50 & 4.00 & $\begin{array}{l}\text { Health } \\
\text { Portal }\end{array}$ & Patients/public & $\begin{array}{l}\text { Update } \\
\text { frequency not } \\
\text { available }\end{array}$ \\
\hline Endocrineweb & https://www.endocrineweb.com & 65.00 & 4.50 & Professional & $\begin{array}{l}\text { Healthcare providers, } \\
\text { patients/public }\end{array}$ & $\begin{array}{l}\text { Website is } \\
\text { updated } \\
\text { occasionally }\end{array}$ \\
\hline $\begin{array}{l}\text { Everyday } \\
\text { Health }\end{array}$ & $\begin{array}{l}\text { https://www.everydayhealth. } \\
\text { com/ }\end{array}$ & 69.00 & 4.00 & $\begin{array}{l}\text { Health } \\
\text { Portal }\end{array}$ & Patients/public & $\begin{array}{l}\text { Update } \\
\text { frequency not } \\
\text { available }\end{array}$ \\
\hline Healthline & https://www.healthline.com/ & 67.50 & 4.00 & $\begin{array}{l}\text { Health } \\
\text { Portal }\end{array}$ & $\begin{array}{l}\text { Healthcare providers, } \\
\text { patients/public }\end{array}$ & $\begin{array}{l}\text { Update } \\
\text { frequency not } \\
\text { available }\end{array}$ \\
\hline Totally Health & https://www.totallyhealth.com & 66.50 & 4.00 & $\begin{array}{l}\text { Health } \\
\text { Portal }\end{array}$ & $\begin{array}{l}\text { Healthcare providers, } \\
\text { patients/public }\end{array}$ & $\begin{array}{l}\text { Update } \\
\text { frequency not } \\
\text { available }\end{array}$ \\
\hline
\end{tabular}

intention of self-diagnosis and seeking treatment options for their health conditions [17], and patients with T2DM searching for information about CAM are no exception [7]. It is important for healthcare providers to be aware of the quality of consumer health information presented on these websites, as this can better allow them to engage in discussions with their patients regarding what constitutes a high-quality online resource and to facilitate shared decision-making.

Of the 480 websites that were identified, 30 met the eligibility criteria and were included in the present study. Across all eligible websites, the mean summed DISCERN score was $52.40(\mathrm{SD}=12.11)$, and the mean overall quality score (question 16$)$ was $3.55(\mathrm{SD}=0.86)$. The total mean scores of questions $2,3,9,10$ and 14 across all assessed websites exceeded four, while all 30 websites scored a mean of below 4 for the remaining questions. Eleven out of the 30 websites scored an overall DISCERN score of 60 or higher. It was found that variability in the quality of information existed across the eligible websites, based on our DISCERN instrument appraisals.
The majority of the eligible websites scored well on the reliability section of the DISCERN instrument, however, it should be noted that one general trend included that low quality sources, as defined by the DISCERN instrument, were referenced by some websites. In contrast, many of the websites scored poorly in the quality of the information section of the DISCERN instrument. A common trend, especially across the websites categorized as professional or health portal, included the highlighting of the academic titles/degrees held by website authors, despite the provision of biased or unreliable information, which in combination could be misleading to patients. Websites providing high-quality information should instead be based upon evidence informed by the medical literature, such as clinical practice guidelines $[18,19]$.

Our findings can be compared to studies which assessed consumer health information on similar topics. Weitzman et al. examined the quality of information available on social networking websites regarding diabetes mellitus in 2010. They found that many of the 
websites they assessed contained misinformation about diabetes treatments, including the provision of unfounded 'cures'; only 30\% of the websites they assessed were considered to be of high quality with respect to the content and information presented [20]. They also reported that many of the online resources available for T2DM lacked disclaimers and failed to communicate to patients the importance of discussing treatment options with their physicians [20]. Another study conducted in 2019 by Kloosterboer et al. assessed the quality of information available online for patients with regards to diabetic retinopathy using JAMA benchmarks [21]. They concluded that the quality of information present online for diabetic retinopathy was both low and difficult for patients to interpret [21]. Another study conducted a content analysis of popular American diabetes websites and concluded that while they were engaging to readers, they often lacked credibility and in-depth information for caregivers and providers [22]. Conclusions derived from previous literature indicate that the quality of information found online surrounding the topic of T2DM and diabetes in general is low and mirrors our findings which found that the quality of CAM-specific T2DM online information is also low.

With respect to the total means of individual DISCERN question scores across all eligible websites, the lowest total mean scores were observed for questions 8 , 11 and 12. These questions assessed the following characteristics respectively: the validity of evidence presented for therapies within the websites; whether the website authors included appropriate assessments of the risks of each therapy suggested within the website; and whether the website authors thoroughly present the implications for choosing not to use a treatment for the disease or condition. Based on our subset of websites assessed, some common indicators that a website contains low quality information included: the website presenting therapy options for a disease or condition without citing supporting scientific evidence, failing to present the health impacts or risks of using a specific therapy for a health condition, or failing to present the implications of choosing not to use treatments or therapies for a health condition.

The present study's findings can also be compared to the literature written about the quality of CAM-related consumer health information. A study conducted by Chen et al. in 2018 identified that the quality of information presented on complementary and integrative health websites was variable; many websites scored well in measures of ownership and navigability, but scored poorly on measures related to source attribution and authorship, bringing into question the reliability of sources used [23]. In the study, Chen et al. concluded that the wide range of website types showed that the public experiences information through a variety of media and it would be helpful to investigate how the presentation of this content varies depending on the medium [23]. Bessel et al. used the DISCERN instrument to assess the quality of information presented online for nonprescription and complementary medicines [24]. They found that consumers that use online resources to collect information about non-prescription and complementary medicines had insufficient access to reliable information and were unable to make informed health decisions about the medications they sought [24]. Three additional studies assessed the quality of online consumer health information at the intersection of CAM and low back pain, arthritis, and neck pain, respectively using the DISCERN instrument, finding that the quality of information was variable across different websites [25-27].

Due to the high prevalence of CAM use in patients with T2DM [28], the discussion between practitioner and patient regarding the safe and effective use of such therapies holds great importance [29]. The vast amount of information present online may present a burden to patients seeking to make informed decisions about their own health [29]. A study found that patient perceptions of control over their health accounted for increased trust in the patient-physician relationship. Trust was higher when patients felt that they had control over their health and perceived communication was participative [30]. Another study found that a high proportion of T2DM patients failed to discuss their use of CAM with their physicians [31]. The present study's findings highlight the importance for healthcare providers to be aware of the quality and reliability of information online regarding CAM treatment options for T2DM, as this can help guide their discussions with their patients. Healthcare providers should be aware of their patients' informationseeking behaviour, assist them in navigating through the content they encounter online, and provide them with resources containing trustworthy and reliable information. Furthermore, a need exists for researchers, healthcare providers and educators to develop guidelines that address these recent and emerging issues associated with online patient health information, raise awareness around online misinformation, and assist patients in identifying reliable online resources [32-35].

\section{Strengths and limitations}

One strength of our study included the use of the DISCERN instrument, which has been found to be valid and reliable in assessing the quality of consumer health information. Additionally, we pilot tested both the data extraction and the application of the DISCERN instrument, which improved the standardization of reporting and inter-rater reliability. Another strength 
includes the fact that we searched four countries across Google, which allows our findings to be more applicable across different jurisdictions. A limitation of this study was the cross-sectional nature of the study; information online is subject to continual change, thus our study only captured the quality of information on this health topic at a snapshot in time. Lastly, because we also only included and assessed English-language websites, our study's findings may not necessarily be generalizable to the quality of information written in other languages online.

\section{Conclusion}

The internet provides an avenue for the rapid dissemination of consumer health information. Patients with T2DM often search for such information online, including that which pertains to CAM and T2DM, and may use this information to guide the decisions they make about their personal health. The purpose of this study was to identify and assess the quality of information on websites providing CAM consumer health information for the treatment and/or management of T2DM. Following the quality assessment of eligible websites using the DISCERN instrument, it was found that, in general, our subset contained low quality consumer health information; most notably, they often failed to provide adequate references to support their health statements. Healthcare providers attending to patients with T2DM should be aware of the low quality of such websites in order to better discuss and promote shared decision-making with patients inquiring about or using CAM.

\section{Abbreviations \\ CAM: complementary and alternative medicine; T2DM: type 2 diabetes mellitus}

\section{Acknowledgements}

JYN was awarded a Research Scholarship and an Entrance Scholarship from the Department of Health Research Methods, Evidence and Impact, Faculty of Health Sciences at McMaster University. We gratefully acknowledge Saif Ali for his assistance with data collection.

\section{Authors' contributions}

JYN: conceptualized and designed the study, collected the data, interpreted and analysed the data, provided contributions and critically revised the manuscript, and gave final approval of the version to be published. MN: interpreted and analysed the data, drafted the manuscript, and gave final approval of the version to be published. KG: collected the data, interpreted and analysed the data, provided contributions and critically revised the manuscript, and gave final approval of the version to be published. All authors have read and approved the manuscript.

\section{Funding}

This study was unfunded.

\section{Declarations}

Ethics approval and consent to participate

This study involved a search and review of publically available online information only; it did not require ethics approval or consent to participate.

\section{Consent for publication}

All authors consent to this manuscript's publication.

\section{Competing interests}

The authors declare that they have no competing interests.

Received: 1 July 2021 Accepted: 17 August 2021

Published online: 17 September 2021

\section{References}

1. International Diabetes Federation. Diabetes facts and figures. 2020 [cited 2021 Feb 10]. Available from: https://www.idf.org/aboutdiabetes/what-is-dia betes/facts-figures.html

2. Chen L, Magliano DJ, Zimmet PZ. The worldwide epidemiology of type 2 diabetes mellitus-present and future perspectives. Nat Rev Endocrinol. 2012;8(4):228-36. https://doi.org/10.1038/nrendo.2011.183.

3. Goyal R, Jialal I. Diabetes mellitus type 2: StatPearls Publishing; 2020. http:// www.ncbi.nlm.nih.gov/books/NBK513253/

4. Hantzidiamantis PJ, Lappin SL. Physiology, glucose: StatPearls Publishing; 2020. http://www.ncbi.n/m.nih.gov/books/NBK545201/

5. Ganesan K, Rana MB, Sultan S. Oral hypoglycemic medications: StatPearls Publishing; 2020. Available from: http://www.ncbi.nlm.nih.gov/books/NBK4 82386/

6. $\mathrm{Ng} J \mathrm{Y}$, Verma KD. Identifying the quantity and assessing the quality of clinical practice guidelines for the treatment and management of type 2 diabetes: a systematic review. Diabetes Res Clin Pr. 2020;7:108365. https:// doi.org/10.1016/j.diabres.2020.108365.

7. Posadzki P, Watson LK, Alotaibi A, Ernst E. Prevalence of use of complementary and alternative medicine (CAM) by patients/consumers in the UK: systematic review of surveys. Clin Med. 2013;13(2):126-31. https:// doi.org/10.7861/clinmedicine.13-2-126.

8. Esmail N. Complementary and alternative medicine: use and public attitudes 1997, 2006, and 2016. Vancouver: Fraser Institute; 2017. Available at: https://www.fraserinstitute.org/sites/default/files/complementary-and-a Iternative-medicine-2017.pdf

9. Ng JY, Boon HS, Thompson AK, Whitehead CR. Making sense of "alternative", "complementary", "unconventional" and "integrative" medicine: exploring the terms and meanings through a textual analysis. BMC Complement Altern Med. 2016;16(1):134. https://doi.org/10.1186/s12906-01 6-1111-3.

10. National Center for Complementary and Integrative Health. Complementary, alternative, or integrative health: what's in a name? 2018 [cited 2021 Feb 10]. Available from: https://www.nccih.nih.gov/health/complementary-a Iternative-or-integrative-health-whats-in-a-name

11. Fan KW. National center for complementary and alternative medicine website. J Med Libr Assoc. 2005;93(3):410-2 [cited 2021 Feb 10]. Available from: https://www.ncbi.nlm.nih.gov/pmc/articles/PMC1176230/.

12. Diabetes and Dietary Supplements. National Center for Complementary and Integrative Health. 2018 [cited 2021 Feb 10]. https://www.nccih.nih.gov/hea Ith/diabetes-and-dietary-supplements

13. Grossman LD, Roscoe R, Shack AR. Complementary and alternative medicine for diabetes. Can J Diabetes. 2018;42:S154-61. https://doi.org/10.1 016/j.jcjd.2017.10.023.

14. Sharma V, Holmes JH, Sarkar IN. Identifying complementary and alternative medicine usage information from internet resources: a systematic review. Method Inf Med. 2016;55(4):322-32. https://doi.org/10.3414/ME15-01-0154

15. StatCounter Global Stats. Search Engine Market Share Worldwide: StatCounter Global Stats; 2020. [cited 2020 Aug 6]. Available from: https://gs. statcounter.com/search-engine-market-share/all/

16. Charnock D. The DISCERN handbook: quality criteria for consumer health information on treatment choices: Radcliffe Medical: University of Oxford and The British Library; 1998. [cited 2021 Feb 10]. Available from: http:// www.discern.org.uk/discern.pdf 
17. Young $\mathrm{N}$, Lotterman $\mathrm{S}$, Simonson E. Patient internet health resource utilization prior to emergency department visits. Am J Emerg Med. 2018;36(10):1911-3. [cited 2021 May 12]. https://doi.org/10.1016/j.ajem.2018.02.032.

18. Chen X, Hay UL, Waters EA, Kiviniemi MT, Biddle C, Schofield E, et al. Health literacy and use and trust in health information. J Health Commun. 2018; 23(8):724-34. https://doi.org/10.1080/10810730.2018.1511658.

19. Ng JY, Verma KD, Gilotra K. Quantity and Quality of Complementary and Alternative Medicine Recommendations in Clinical Practice Guidelines for Type 2 Diabetes Mellitus: A Systematic Review. Nutrition Metabolism and Cardiovascular Diseases. 2021. https://doi.org/10.1016/j.numecd.2021.07.029

20. Weitzman ER, Cole E, Kaci L, Mandl KD. Social but safe? Quality and safety of diabetes-related online social networks. J Am Med Inform Assn. 2011;18(3): 292-7. https://doi.org/10.1136/jamia.2010.009712.

21. Kloosterboer A, Yannuzzi NA, Patel NA, Kuriyan AE, Sridhar J. Assessment of the quality, content, and readability of freely available online information for patients regarding diabetic retinopathy. JAMA Ophthalmol. 2019;137(11): 1240-5. https://doi.org/10.1001/jamaophthalmol.2019.3116.

22. Holtz BE. Evaluating the most popular diabetes websites in the USA: a content analysis. Health Promot Int. 2020;35(6):1394-405. https://doi.org/1 0.1093/heapro/daaa008.

23. Chen AT, Taylor-Swanson L, Buie RW, Park A, Conway M. Characterizing websites that provide information about complementary and integrative health: systematic search and evaluation of five domains. Interact J Med Res. 2018;7(2):e14. https://doi.org/10.2196/ijmr.9803.

24. Bessell TL, Anderson JN, Silagy CA, Sansom LN, Hiller JE. Surfing, selfmedicating and safety: buying non-prescription and complementary medicines via the internet. BMJ Qual Saf. 2003;12(2):88-92. https://doi.org/1 $0.1136 /$ ghc. 12.2 .88 .

25. Ng JY, Gilotra K. Web-information surrounding complementary and alternative medicine for low Back pain: a cross-sectional survey and quality assessment. Integr Med Res. 2020;18(3):100692. https://doi.org/10.1016/j. imr.2020.100692.

26. Ng JY, Vacca A, Jain T. The quality of online consumer health information at the intersection of complementary and alternative medicine and arthritis. Adv Rheumatol. 2021;61(1):1-7. https://doi.org/10.1186/s42358-021-00162-y.

27. Ng JY, Saini JB, Dzisiak DA. Evaluating the quality of websites providing complementary and alternative medicine patient information for neck pain. Advances in Integrative Medicine. 2021. https://doi.org/10.1016/j.aimed.2 021.05.001.

28. Candar A, Demirci H, Baran AK, Akpınar Y. The association between quality of life and complementary and alternative medicine use in patients with diabetes mellitus. Complement Ther Clin. 2018;31:1-6. https://doi.org/10.101 6/j.ctcp.2018.01.002.

29. Gantenbein L, Navarini AA, Maul LV, Brandt O, Mueller SM. Internet and social media use in dermatology patients: search behavior and impact on patient-physician relationship. Dermatol Ther. 2020;29(6):e14098. https://doi. org/10.1111/dth. 14098 .

30. Gabay G. Perceived control over health, communication and patientphysician trust. Patient Educ Couns. 2015;98(12):1550-7. https://doi.org/10.1 016/j.pec.2015.06.019.

31. Huri HZ, Lian GTP, Hussain S, Pendek R, Widodo RT. A survey amongst complementary alternative medicine (CAM) users with type 2 diabetes. Int J Diabetes Metab. 2009;17(1):9-15 Available from: https://vlibrary.emro.who. int/imemr/a-survey-amongst-complementary-alternative-medicine-ca musers-with-type-2-diabetes-2/.

32. Diviani N, van den Putte B, Giani S, van Weert JC. Low health literacy and evaluation of online health information: a systematic review of the literature. J Med Internet Res. 2015;17(5):e112. https://doi.org/10.2196/jmir.4018.

33. Pleasant A, McKinney J. Coming to consensus on health literacy measurement: an online discussion and consensus-gauging process. Nurs Outlook. 2011:59(2):95-106. https://doi.org/10.1016/j.outlook.2010.12.006.

34. Weis BD. Health literacy: a manual for clinicians: American Medical Foundation; 2007. [cited 2021 May 18]. Available from: http://www.pa rtnershiphp.org/Providers/HealthServices/Documents/Health\%20Education/ CandLToolKit/2\%20Manual\%20for\%20Clinicians.pdf

35. National Institutes of Health. How to write easy-to-read health materials. Bethesda: National Library of Medicine; 2013. [cited 2021 May 18]

\section{Publisher's Note}

Springer Nature remains neutral with regard to jurisdictional claims in published maps and institutional affiliations.

\section{Ready to submit your research? Choose BMC and benefit from}

- fast, convenient online submission

- thorough peer review by experienced researchers in your field

- rapid publication on acceptance

- support for research data, including large and complex data types

- gold Open Access which fosters wider collaboration and increased citations

- maximum visibility for your research: over $100 \mathrm{M}$ website views per year

At BMC, research is always in progress.

Learn more biomedcentral.com/submissions 